\title{
EL “SE” TÉLICO Y LA DELIMITACIÓN DEL COMPLEMENTO VERBAL EN EL ESPAÑOL DE ARGENTINA Y DE VENEZUELA
}

Jean Carlos da Silva GOMES (D) $\boldsymbol{\Delta}$

Universidade Federal do Rio de Janeiro (UFRJ)

ఠ

OPEN ACCESS

EDITADO POR

- Miguel Oliveira, Jr. (UFAL)

- René Almeida (UFS)

REVISADO POR

- Roberta Oliveira (UFSC)

- Teresa Wachowicz (UFPR)

- Raquel Lawall (UFJF)

SOBRE LOS AUTORES

- Jean Carlos da Silva Gomes Conceptualización, Escritura -

Versión Original y Escritura

- Análisis y Edición.

- Adriana Leitão Martins

Conceptualización, Escritura -

Versión Original y Escritura

- Análisis y Edición.

\section{FECHAS}

- Recebido: 03/02/2020

- Aceito: 16/06/2020

- Publicado: 28/11/2020

\section{COMO CITAR}

GOMES, Jean Carlos da Silva; MARTINS, Adriana Leitão (2020). El "se" télico y la delimitación del complemento verbal en el español de Argentina y de Venezuela. Cadernos de Linguística, v. 1, n. 2, p. 01-23.

\section{Adriana Leitão MARTINS (iD $\boldsymbol{\Delta}$ \\ Universidade Federal do Rio de Janeiro (UFRJ)}

\section{RESUMEN}

La telicidad es una noción aspectual semántica que se caracteriza por la marcación lingüística en la oración de un punto final inherente de la situación. Según Moure (1990), en el complemento verbal, la presencia de determinantes singulares conduce a una lectura télica de la oración mientras que la ausencia de determinantes conduce a una lectura atélica, pero no se sabe a qué lectura conduce la presencia de determinantes plurales. En español, hay un clítico que se conoce por 'se' télico que solamente se puede usar en oraciones que transmiten el valor aspectual de telicidad. Por un lado, De Miguel (1999) afirma que la presencia de determinantes plurales indefinidos en el complemento verbal conduce a una lectura atélica y, por eso, no se permite el uso del 'se' télico en oraciones que contengan dicha construcción, al paso que, para Gomes y Martins (2020), basados en datos del español de España, esa construcción verbal conduce a una lectura télica y, por lo tanto, el uso del setélico es posible. Así, se objetiva en este trabajo verificar si se puede combinar el se télico con verbos cuyos complementos sean introducidos por determinantes plurales indefinidos en el español de Argentina y de Venezuela. Para este fin, se aplicó un test de juicio de gramaticalidad 
comentado a 15 hablantes de cada una de esas variedades. Los resultados indican que dicha combinación es posible en ambas. Se discutió que el valor aspectual télico de una oración no depende de una cuantificación precisa para que se delimite el complemento.

\section{ABSTRACT}

Telicity is an aspectual semantic notion characterized by the presence in the sentence of a linguistically marked inherent endpoint of the situation. According to Moure (1990), the presence of singular determiners in the verbal complement leads to a telic reading of the sentence while the absence of determiners in the verbal complement leads to an atelic reading, but it is not known to which reading the presence of plural determiners in the verbal complement leads. In Spanish, there is a particle known as telic se that appears only in sentences that carry the telic aspectual value. De Miguel (1999) states that the presence of indefinite plural determiners in the verbal complement leads to an atelic reading and, due to that, the telic se cannot appear in sentences with such verbal construction. Gomes and Martins (2020), based on Spanish spoken in Spain data, state that this verbal construction leads to a telic reading and the telic se appears in sentences with such structure. In view of this, the objective of this work is to verify if the telic se can be combined with verbs whose complements are introduced by indefinite plural determiners in Argentine and VenezueIan Spanish. For that, a commented grammaticality judgment test was applied to 15 speakers of each one of these varieties. The results indicated that this combination is possible both in Argentine and Venezuelan Spanish. We argue that the telic aspectual value of a sentence does not depend on a precise quantification for delimiting the complement.

\section{PALAVRAS-CHAVE}

Telicidad; Determinantes Plurales Indefinidos; Español; Argentina; Venezuela.

\section{KEYWORDS}

Telicity; Indefinite Plural Determiners; Spanish; Argentina; Venezuela. 


\section{INTRODUÇÃO}

La marcación lingüística de un punto final inherente de la situación en la oración es lo que caracteriza la noción aspectual semántica de telicidad (COMRIE, 1976; DAHL, 1977; DECLERCK, 1991; SLABAKOVA, 2000; BERTINETTO, 2001; BASSO, 2007). Una de las maneras de realizar esa delimitación es por medio de un complemento directo delimitado (DE MIGUEL, 1999; LOURENÇONI, 2014; GOMES; MARTINS, 2020), como en "Lucía leyó el libro".

Con relación a la delimitación del complemento verbal, se puede destacar la importancia que tienen los determinantes en la expresión del punto final inherente de la situación. Moure (1990) señala que, en español, la presencia de determinantes singulares como "el / un" en el complemento verbal, como en el ejemplo presente en el párrafo anterior, conduce a una lectura télica, mientras que la ausencia de determinantes en el complemento verbal, como en "Lucía leyó libros", conduce a una lectura atélica, pero no se puede establecer a qué lectura conducen los complementos verbales introducidos por determinantes plurales, como en "Lucía leyó unos libros".

En cuanto a los determinantes plurales, autores como De Miguel (1999) apuntan que, en español, la presencia en el complemento verbal de indefinidos como "unos / algunos / muchos / varios", que se denominan en este artículo como determinantes plurales indefinidos, conduce a una lectura atélica de la oración. En cambio, autores como Gomes y Martins (2020), al analizar datos lingüísticos del español de España, defienden que la presencia de esos determinantes en el complemento verbal conduce a una lectura télica.

En español, hay un clítico, que se conoce por "se" télico, que figura solamente en oraciones télicas, como en "Lucía se leyó un libro". Teniendo en cuenta que, para De Miguel (1999), los determinantes plurales indefinidos, cuando introducen el complemento verbal, conducen a una lectura télica, es posible afirmar que, para esa autora, ese clítico no puede asociarse a verbos cuyos complementos sean introducidos por esos determinantes, al paso que, para Gomes y Martins (2020), dicha asociación es posible en el español de España. Luego, para De Miguel (1999), una oración como "Lucía se leyó algunos libros" es agramatical, mientras que, para Gomes y Martins (2020), no lo es.

Así, en este trabajo, se objetiva contribuir a la comprensión de lo que caracteriza la telicidad. De manera específica, se pretende investigar la compatibilidad del "se" télico con verbos cuyos complementos sean introducidos por determinantes plurales indefinidos en el español de Argentina y de Venezuela. Se considera que dicha investigación permite el planteamiento de una discusión acerca de la contribución de los determinantes plurales indefinidos para el valor aspectual de telicidad en la oración.

La hipótesis que se adopta en este estudio es la de que se puede usar el "se" télico combinado con verbos cuyos complementos sean introducidos por determinantes plurales 
indefinidos en el español de Argentina y de Venezuela. Su formulación se basa en los resultados descritos por Gomes y Martins (2020).

En el intento de someter a prueba la hipótesis de este estudio, se aplicó un test lingüístico de juicio de gramaticalidad comentado, desarrollado por Gomes y Martins (2020), a quince hablantes de español de Argentina y a quince de Venezuela. La tarea consistía en juzgar oraciones que contenían el "se" télico combinado con un verbo cuyo complemento era introducido por un determinante plural indefinido como naturales o raras. Además, los participantes deberían hacer los cambios necesarios para transformar las oraciones que consideraran raras en naturales. Así, si los participantes las juzgaran como raras e hicieran cambios con respecto a la presencia del "se" télico o del determinante utilizado, se podría refutar la hipótesis.

Este artículo está organizado de la siguiente manera: en la segunda sección, se describe la noción aspectual de telicidad; en la tercera sección, se presentan sus formas de realización lingüística en español; en la cuarta sección, se explica la metodología empleada en este trabajo; en la quinta sección, se exponen los resultados de esta investigación; en la sexta sección, se presenta un análisis de los resultados; y, por fin, en la última sección, se expone la conclusión del estudio.

\section{LA NOCIÓN ASPECTUAL SEMÁNTICA DE TELICIDAD}

Aspecto, según Comrie (1976), se define como las diferentes maneras de visualizar la composición temporal interna de una situación. Se asuma que el aspecto en general se caracteriza como una información lingüística que se obtiene composicionalmente (SMITH, 1991; DE MIGUEL, 1999; CELERI, 2008). Por un lado, el aspecto gramatical se define a partir de elementos sintácticos, como la morfología verbal, como es el caso del español, por otro lado, el aspecto semántico ${ }^{1}$ se define a partir de determinados valores semánticos inherentes a la raíz verbal, a los argumentos y/o a los adjuntos presentes en la oración. En este trabajo, se enfoca el estudio del aspecto semántico.

La telicidad es la información relacionada al aspecto semántico que se entiende como la marcación lingüística de un punto final inherente de la situación en la oración (COMRIE, 1976; DAHL, 1977; DECLERCK, 1991; SLABAKOVA, 2000; BERTINETTO, 2001; BASSO, 2007). Luego, una oración télica se caracteriza como aquella que contiene esa marcación

1 En la literatura acerca del aspecto en la lengua española, se utiliza comúnmente el término "aspecto léxico" para referirse a lo que se describe en este artículo como aspecto semántico. Sin embargo, se considera que el uso de la nomenclatura "aspecto léxico" puede conducir a la interpretación de que solo los rasgos del verbo serían suficientes para su expresión. Se asume en este trabajo que otros componentes oracionales también contribuyen a la expresión lingüística del valor aspectual semántico, así que se considera más adecuado el uso de este término. 
mientras que una oración atélica no la contiene. Para que se comprenda dicha diferencia, se presenta a continuación un análisis de los ejemplos (1) y (2).

(1) María comió una manzana.

(2) María comió manzanas.

El ejemplo (1) contiene una oración télica delimitada por el complemento "una manzana" y, por eso, posee la marcación lingüística de un punto final delimitado, de manera que se interpreta por medio de esa oración que la acción de "comer" no puede continuar más allá de ese punto. Por otro lado, el ejemplo (2) contiene una oración atélica que no posee la marcación lingüística de un punto final delimitado por el complemento "manzanas".

Aunque en los ejemplos (1) y (2) el análisis acerca del valor aspectual de telicidad de la oración se haya basado en las características del complemento verbal, se puede destacar que esa no es la única manera posible de hacerlo. Otros elementos oracionales también contribuyen a la lectura télica de la oración, como adjuntos preposicionados (WACHOWICZ, 2008) y sujetos verbales (VERKUYL, 2005) que confieren lingüísticamente un punto final a la situación que se denota por el verbo, como se ejemplifica, respectivamente, en (3) y (4) a continuación.

(3) Juan nadó hasta el fin de la piscina.

(4) El niño vendió su libro.

Respecto a la integración de los rasgos de diferentes constituyentes oracionales en la generación del valor de telicidad, Verkuyl (2005) señala que uno de los rasgos que contribuyen a la caracterización de las situaciones es el $[ \pm S Q A]$ de los sintagmas nominales (SNs), relacionado a la cuantificación de los ítems presentes en esos sintagmas, tanto en posición de complemento como de sujeto. La sigla SQA proviene del término en inglés "Specified Quantity of A" y, por lo tanto, se refiere a la especificación de la cantidad de ítems que se denota por medio del determinante que introduce el SN.

Ese rasgo, cuando marcado positivamente, denota una cantidad específica de cosas o masa. Cuando una situación no es un estado, la especificación de ese rasgo en el sujeto y en el complemento es responsable por el valor de telicidad de la oración. Así, cuando marcado positivamente en ambos ítems, se considera la oración con un verbo dinámico como télica y, cuando negativamente en uno o más, se la considera atélica.

Para el autor, la oración "Mary walked three miles" (María caminó tres millas) es télica, pues tanto el SN sujeto como el SN objeto contienen el rasgo [+SQA]. Por otro lado, la oración 
"Children walked three miles" (Niños caminaron tres millas) es atélica, pues, aunque el SN en posición de complemento contenga el rasgo [+SQA], el SN sujeto posee el rasgo [-SQA]. Así, la oración presente en (4) también podría considerarse télica.

Aunque existan distintas maneras de verificar el valor aspectual semántico de telicidad de la oración, se debe considerar que el análisis del tipo de complemento presente en el predicado verbal es una de las maneras más relevantes de comprender ese fenómeno (BERTINETTO, 2001). Moure (1990), por ejemplo, propuso un continuum, que se conoce como grado de definición del complemento, en el que representa la relación entre el tipo de determinante que introduce el complemento verbal y su contribución con la lectura aspectual de telicidad de la oración, como se puede verificar en la imagen 1, a continuación.

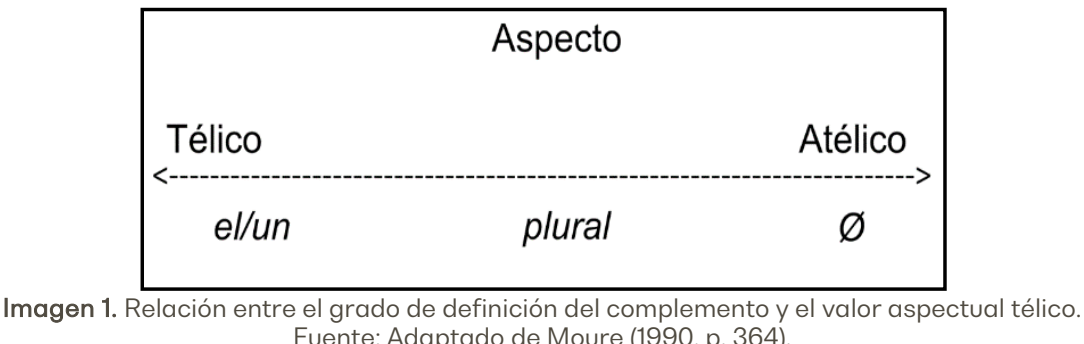

En su propuesta, Moure (1990) señala que la presencia de determinantes singulares como "el / un" en el complemento verbal, como se ve en el ejemplo (5), conduce a una lectura télica de la oración y, por eso, ocupan una posición a la izquierda del continuum, mientras que la ausencia de determinantes en el complemento verbal, como en los nombres escuetos, por ejemplo en (6), conduce a una lectura atélica y, por eso, el $\varnothing$ ocupa una posición a la derecha del continuum. Sin embargo, no se puede establecer a qué lectura, télica o atélica, conducen los determinantes plurales en el complemento verbal, como se ejemplifica en (7).

(5) Juan bebió el / un zumo.

(6) Juan bebió zumos.

(7) Juan bebió unos / algunos / muchos / varios zumos.

Se considera que determinantes como "unos(as) / algunos(as) / muchos(as) / varios(as)" son determinantes plurales indefinidos cuyo complemento es un SN. Dichos determinantes son artículos o pronombres que no son capaces de conferirle una cuantificación precisa al complemento, es decir, su significado no remite a un número exacto de constituyentes abarcados en el SN que los sigue. En este trabajo, se enfoca comprender el papel que los 
determinantes plurales indefinidos desempeñan en la transmisión del valor aspectual de telicidad de una oración.

Verkuyl (2005) señala que complementos directos con cuantificación genérica conducen a una lectura atélica de la oración. Como determinantes plurales indefinidos que introducen complementos verbales no confieren una cuantificación precisa al nombre presente en el complemento, se puede interpretar que, para ese autor, esos determinantes ocuparían una posición a la derecha del continuum presentado por Moure (1990).

Por otra parte, Rothstein (2008), al analizar datos lingüísticos del inglés, indica que el complemento del verbo que conduce a una lectura télica de la oración no depende de la expresión exacta de cantidad de lo que se mide en el complemento, pues, aunque no sea precisa, hay en este constituyente una expresión de cantidad suficiente para delimitar el sintagma verbal. Para ilustrar esa idea, entre los ejemplos de oraciones télicas que ofrece Rothstein (2008, p. 4), se destaca el que contiene el determinante referido en este artículo como plural indefinido: "John ate a lot of chocolates in half an hour" ("Juan comió muchos chocolates en media hora”). Luego, para esa autora, los determinantes plurales indefinidos ocuparían la posición a la izquierda del continuum propuesto por Moure (1990).

\section{TELICIDAD EN ESPAÑOL}

En español, es posible realizar lingüísticamente la noción aspectual de telicidad por medio de tres maneras (DE MIGUEL, 1999; LOURENÇONI, 2014; GOMES; MARTINS, 2020). La primera se caracteriza por el uso de un complemento directo delimitado, como se ejemplifica en (8), descrito como aquel que confiere un límite al evento. La segunda se da por medio de un sintagma preposicional delimitador, como se ve en el ejemplo (9). La tercera manera es por medio del uso del "se" télico combinado con un verbo que contenga un complemento directo delimitado, como se ejemplifica en (10).

(8) Pedro comió una galleta.

(9) Pedro caminó hasta la salida de la escuela

(10) Pedro se comió una galleta.

Se destaca que el uso del "se" télico solo es posible cuando combinado con un verbo cuyo complemento directo sea delimitado, como se ejemplifica en (10). Por otro lado, la presencia del "se" télico combinado con un verbo cuyo complemento directo no contenga un determinante, como en nombres escuetos, genera una agramaticalidad en la oración, como se ejemplifica en (11). 
(11) *Pedro se comió galleta.

Se encuentran en la literatura otras nomenclaturas para referirse al "se" télico, como "se" aspectual (LÓPEZ, 2002), clítico télico (SANZ; LAKA, 2002), partícula "se" (GOMES, 2016) y operador aspectual "se" (LOURENÇONI, 2017). En este trabajo, se optó por utilizar el término "se" télico con base en los estudios de De Miguel (1999), Martins, Gomes y Lourençoni (2017) y Gomes y Martins (2020). Además, se debe destacar que el término "se" télico se refiere a todas las otras formas que ese clítico pueda asumir ("me", "te", "se", "nos" y “os"), debido a que, en la oración, concuerda en número y persona con el sujeto verbal.

La presencia de ese clítico en las frases es opcional en el español y, por eso, no es el responsable por conferirle el valor de telicidad a la oración, sino que, en realidad, lo confirma, puesto que su uso solo es posible en enunciados que sean télicos, cuya delimitación ya haya sido conferida por medio de otros constituyentes oracionales. Así, se interpreta que el valor aspectual de telicidad del clítico "se" está subespecificado en una oración. Es decir, son los otros elementos que la constituyen que determinan su valor télico de manera que la especificación del clítico ocurre después de la concatenación de los ítems léxicos de la oración.

Algunos contextos favorecen el uso del "se" télico. Uno de ellos es la presencia de verbos de ingestión (SANZ, 2000), definidos como aquellos cuyo significado remite a la idea de que el sujeto toma algo para sí (ARCE ARCENALES, 1989 apud LÓPEZ, 2002), como "comer", "beber", "tomar" etc. En el ejemplo (10), presente en el inicio de la sección, se puede verificar la combinación del "se" télico con el verbo de ingestión "comer".

D’Introno, González y Rivas (2007), al discutir sobre el uso del "se" en español, interpretándolo como un dativo reflexivo de interés, señalan que, combinado con verbos de ingestión, puede asumir el valor semántico de benefactivo. Otra interpretación posible es la de completud del evento, o sea, se entiende que la acción se ha realizado completamente. Bajo esta perspectiva, una frase como la que se presenta en (10) incluye el significado de que Pedro se comió la galleta entera (D’INTRONO; GONZÁLEZ; RIVAS, 2007; SUÁREZ-CEPEDA, 2005).

Más allá de las interpretaciones posibles que la combinación del "se" con verbos de ingestión pueda generar, nos interesa en este trabajo tener en claro que una de sus funciones en la oración es la de confirmar su valor aspectual de telicidad (ROLDÁN, 1971; DE MIGUEL, 1999; DE MIGUEL; LAGUNILLA, 1999; SANZ; LAKA, 2002; LOURENÇONI; MARTINS, 2017).

De Miguel (1999) señala que solo se puede usar ese clítico en oraciones con verbos transitivos, como se observa en el ejemplo (10), o inacusativos, como en (12), recuperado de De Miguel (1999, p. 2996). Además, el uso de la morfología perfectiva parece favorecer la 
presencia del clítico télico (DE MIGUEL; LAGUNILLA, 2000), como se puede verificar también en el ejemplo (10).

(12) El libro se ha caído del estante.

Según Lourençoni y Martins (2016), los contextos en los que se puede usar el "se" télico aún son tema de investigación en la literatura. En este trabajo, se investiga la compatibilidad de ese clítico con verbos cuyos complementos sean introducidos por determinantes plurales indefinidos.

Teniendo en cuenta que ese clítico solo puede figurar en oraciones con valor aspectual télico, como en (10), y no en las que presentan el valor aspectual atélico, como en (11), se utiliza en este trabajo la presencia del "se" como un instrumento seguro para garantizar el valor aspectual de telicidad que transmite la oración. Luego, si la combinación del "se" télico con un verbo cuyo complemento contenga un determinante plural indefinido sea posible, se podrá afirmar que el valor aspectual de ese tipo de determinante contribuye a una lectura télica de la oración. Al paso que si dicha combinación no sea posible, se podrá argumentar que ese tipo de determinante contribuye a una lectura atélica de la oración. Es decir, por medio de los datos obtenidos en este trabajo, se pretende discutir la contribución de determinantes plurales indefinidos para el valor aspectual télico de la oración.

De Miguel (1999) señala que no se puede combinar el "se" télico con verbos cuyos complementos sean introducidos por determinantes plurales indefinidos, debido a que, para esa autora, esos determinantes no son capaces de conferirle un límite al evento. Luego, la frase en el ejemplo (13) sería considerada agramatical en español para esa autora.

(13) Pedro se comió unas galletas.

En cambio, Gomes y Martins (2020), al analizar datos del español de España, constataron que dicha combinación es posible y, de conformidad con Rothstein (2008), sostienen que los determinantes plurales indefinidos, aunque no sean capaces de conferirle una cuantificación precisa al complemento, presentan una delimitación al evento. En consecuencia, la frase en el ejemplo (13), bajo esta perspectiva, sería considerada gramatical.

Otros autores, como Suárez-Cepeda (2005) y Márquez (2020), al discurrir sobre contextos en los que se puede usar el "se" télico, formularon ejemplos en los cuales es posible observar su compatibilidad con verbos cuyos complementos sean introducidos por determinantes plurales indefinidos, como en los ejemplos (14), recuperado de Suárez-Cepeda (2005, p. 279), y (15), recuperado de Márquez (2020, p. 9). Esos autores 
no discutieron en sus trabajos la contribución de esos determinantes en la transmisión del valor de telicidad de la oración, pero es posible interpretar que, como consideran dicha compatibilidad gramatical en español, entienden que determinantes plurales indefinidos conducen a una lectura télica. ${ }^{2}$

(14) Pedro se tomó algunos / unos vinos.

(15) Mi hermano se fumó varios cigarros.

En resumen, se puede afirmar que los expertos se dividen en dos enfoques distintos. Por un lado, autores como Verkuyl (2005) y De Miguel (1999) sugieren que determinantes plurales indefinidos en el complemento verbal conducen a una lectura atélica de la oración, y, así, la presencia del "se" télico la convierte en agramatical. Por otro lado, autores como Rothstein (2008) y Gomes y Martins (2020) sugieren que esos determinantes en el complemento verbal conducen a una lectura télica de la oración y, por eso, la presencia del "se" télico sería gramatical. En este trabajo, se investiga tal cuestionamiento por medio de datos lingüísticos del español de Argentina y de Venezuela.

\section{METODOLOGÍA}

En este trabajo, se aplicó un test lingüístico a treinta personas, divididas en quince hablantes nativos de español de Argentina y quince de Venezuela. El perfil de los participantes era de sujetos con edad entre veinte y cincuenta y cinco años con curso superior completo o incompleto.

Los participantes de Argentina son nativos y residentes de las ciudades de Buenos Aires y Rosario que, según Fontanella de Weinberg (2004), forman parte de la zona dialectal, entre las siete existentes en el país, conocida como Litoral. Los hablantes de Venezuela, a su vez, son nativos y residentes de la ciudad de Caracas.

Se utilizó un test lingüístico de juicio de gramaticalidad comentado off-line desarrollado por Gomes y Martins (2020). La tarea consistía en juzgar enunciados como naturales o raros. Si al participante le pareciera natural el enunciado, debería escribir

2 Se resalta que, en esos trabajos, no se ha realizado una recogida de datos de ninguna variedad de la lengua española. Suárez-Cepeda (2005) desarrolló un estudio sobre adquisición de español como L2, en el que la afirmación sobre la combinación entre el "se" télico y verbos cuyos complementos sean introducidos por determinantes plurales indefinidos está presente en su fundamentación teórica. Márquez (2020), a su vez, desarrolló un estudio que contenía ejemplos de su autoría, en los que se observó dicha combinación. 
"OK", al paso que, si le pareciera raro, debería hacer los cambios necesarios para transformarlo en natural.

Ese método se basa en los moldes propuestos por Chomsky (1957), en los cuales se entiende que se debe juzgar el enunciado desde una perspectiva estructural. Así, se supone que los participantes usaron el conocimiento interno que poseen de su lengua materna para realizar la tarea. Los tests de juicio de gramaticalidad demuestran su eficiencia en distintos ámbitos de la investigación lingüística, como la descripción de lenguas naturales (CHOMSKY, 1957; KRIFKA, 2011), adquisición de L1 (GORDON, 1996; GROLLA, 2009), adquisición de L2 (ZOBL, 1992; GRÉGIS, 2007) e incluso en el estudio del deterioro lingüístico (MARTINS, 2010; WILSON et al., 2010; GOMES, 2020).

Autores como Gass (1979; 1980), Lightbown, Spada y Wallace (1980) y Liceras (1985) defienden que es importante que los participantes no se limiten al juicio del enunciado como gramatical o agramatical, sino que también corrijan los agramaticales, pues esta es una de las evidencias más claras para el entendimiento de lo que causa dicha agramaticalidad. Según Chaudron (2003), ese tipo de test evalúa el conocimiento metalingüístico del participante y por eso forma parte de un subconjunto de tests lingüísticos conocido como "tareas de conocimiento metalingüístico". Además, en algunos trabajos sobre el uso del "se" télico en español, como los de Martins, Gomes y Lourençoni (2017) y Lourençoni y Martins (2017), los resultados de la aplicación de ese test se han mostrado muy eficientes para el estudio del fenómeno lingüístico.

Así, se comprende que la tarea de alterar enunciados para transformarlos en gramaticales hace que clasifiquemos ese test como uno de juicio de gramaticalidad comentado. Ese método permite que el informante busque conscientemente el elemento que hace que el enunciado sea agramatical e intente encontrar una alternativa para volverlo natural. Así, en lo que respecta a este trabajo, es posible averiguar si el problema que identifica el informante en el enunciado reside específicamente en la presencia del "se" télico o del determinante plural indefinido en la posición de complemento verbal combinados en la misma oración.

En el test había doce enunciados, de los cuales cuatro eran enunciados objetivo, correspondiente a $1 / 3$ de los estímulos del test, y ocho distractores, correspondiente a $2 / 3$, modelo frecuentemente utilizado en experimentaciones lingüísticas, como sugieren autores como Havik et al. (2009)3. Todos los enunciados objetivo contenían el "se" télico combinado con un verbo cuyo complemento era introducido por un determinante plural indefinido, como se puede ver en el ejemplo (16).

3 Se implementa esta distribución de estímulos objetivo y distractores en el intento de impedir que los participantes se concienticen de la construcción efectivamente evaluada en el test, lo que podría influenciar en los resultados. Aun así, es importante señalar que no es posible garantizar que ningún participante perciba el enfoque de la experimentación 
(16) Juan se comió algunas manzanas.

Con el objetivo de garantizar que la evaluación acerca de la (a)gramaticalidad de los enunciados se restringiera a la compatibilidad investigada en este estudio, se optó, en los enunciados objetivo, por mantener los contextos que favorecen el uso del "se" télico. Así, se usaron, en esas frases, solamente verbos transitivos, con carácter de ingestión y morfología perfectiva.

De los ocho enunciados distractores, cuatro eran gramaticales y cuatro eran agramaticales. Los enunciados gramaticales contenían periodos bien formulados con un sujeto, un verbo transitivo y un complemento verbal, como en el ejemplo (17). Las frases agramaticales, por otro lado, se caracterizaban por la ausencia del complemento directo en verbos transitivos, como en el ejemplo (18), o por la ausencia de un determinante en el sujeto de la frase, como se ve en (19).

(17) Celina estudió inglés.

(18) *Lucía quiso.

(19) *Animal mató al hombre.

El orden por medio de la cual se presentaron los enunciados a los participantes ha sido pseudoaleatorizado. Como no había una práctica anterior a la realización del test para que los participantes comprendieran la tarea que realizarían, los dos primeros enunciados eran distractores, a fin de que se acostumbraran al modelo del test. Además, en ningún momento, un enunciado objetivo precedía otro enunciado objetivo y, por eso, había, por lo menos, un distractor entre ellos.

Por fin, se aplicó el test por medio de un formulario Google, que se ha enviado a los participantes por internet.

\section{RESULTADOS}

En esta sección, se presentan primeramente los resultados obtenidos por medio de la aplicación del test de juicio de gramaticalidad comentado a los hablantes de Argentina y, enseguida, los de Venezuela. Se destaca que, como cada grupo de quince participantes ha juzgado cuatro enunciados objetivo, se ha obtenido un total de sesenta respuestas para cada variedad. 
En las respuestas de los hablantes nativos de Argentina, en $68 \%$ de los enunciados objetivo (cuarenta y un enunciados), que contenían el "se" télico combinado con un verbo cuyo complemento era introducido por un determinante plural indefinido, no se hicieron modificaciones en lo que respecta a la presencia de un determinante plural indefinido y del "se" télico. Por otro lado, en $32 \%$ de los enunciados objetivo (diecinueve enunciados), los participantes excluyeron el "se" télico y mantuvieron el determinante plural indefinido.

El gráfico 1, a continuación, se trata de un resumen de los resultados obtenidos en la aplicación del test de juicio de gramaticalidad comentado a los participantes hablantes nativos del español de Argentina.

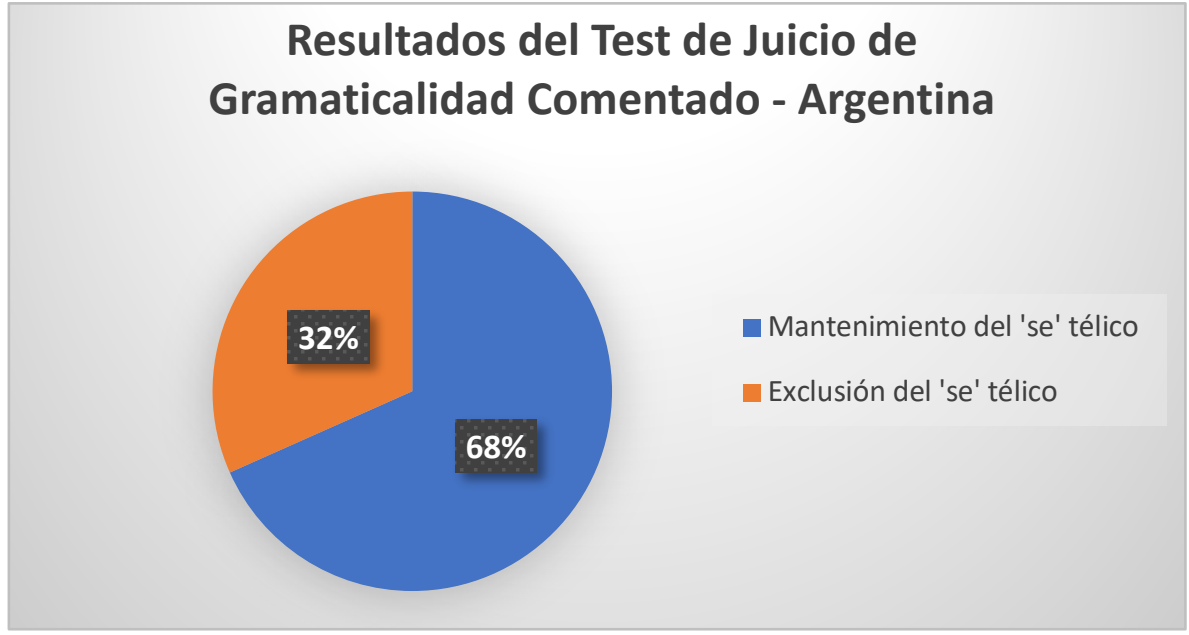

Gráfico 1. Resultados del test de juicio de gramaticalidad comentado - Argentina.

Se percibe que solo $60 \%$ de los enunciados objetivo no sufrieron ninguna modificación, ya que, eventualmente, los participantes alteraban algún ítem del enunciado, pero en ningún caso se ha modificado el determinante plural indefinido por uno de otra naturaleza. Entre las modificaciones que hicieron los participantes, en seis enunciados, se alteró el verbo, como en el ejemplo (20), en el que se sustituyó el verbo "beber" por "tomar"; en dos enunciados, se modificó el determinante plural indefinido por otro determinante plural indefinido, como en el ejemplo (21), en el que se sustituyó "algunos" por "unos"; y, por fin, en un enunciado, se modificó el ítem léxico presente en el complemento verbal, como en el ejemplo (22), en el que se sustituyó el término "cigarros" por "cigarrillos".

(20) María se tomó unos vasos de agua.

(21) Leticia fumó unos cigarros.

(22) Leticia se fumó algunos cigarrillos. 
Por medio de la evaluación del juicio de gramaticalidad de los sujetos en cada enunciado del test separadamente, se observó que la aceptación de la combinación investigada en este trabajo era más amplia con algunos enunciados que con otros, como se ve en el cuadro 1 a continuación.

\begin{tabular}{|c|c|c|c|c|}
\hline Enunciado & $\begin{array}{l}\text { Sin } \\
\text { modificación }\end{array}$ & $\begin{array}{l}\text { Matenimiento del } \\
\text { "se" télico y } \\
\text { modificación en el } \\
\text { enunciado }\end{array}$ & $\begin{array}{l}\text { Exclusión del "se" } \\
\text { télico }\end{array}$ & $\begin{array}{l}\text { Exclusión del "se" } \\
\text { télico y modificación } \\
\text { en el enunciado }\end{array}$ \\
\hline $\begin{array}{l}\text { Juan se comió } \\
\text { algunas } \\
\text { manzanas }\end{array}$ & $53 \%$ & & $\begin{array}{l}\text { Juan comió algunas } \\
\text { manzanas }(47 \%)\end{array}$ & \\
\hline $\begin{array}{l}\text { María se bebió } \\
\text { unos vasos de } \\
\text { agua }\end{array}$ & $33 \%$ & $\begin{array}{l}\text { María se tomó unos } \\
\text { vasos de agua ( } 27 \%)\end{array}$ & $\begin{array}{l}\text { María bebió unos } \\
\text { vasos de agua (27\%) }\end{array}$ & $\begin{array}{l}\text { María tomó unos } \\
\text { vasos de agua (13\%) }\end{array}$ \\
\hline $\begin{array}{l}\text { Miguel se tomó } \\
\text { unas cervezas }\end{array}$ & $80 \%$ & & $\begin{array}{l}\text { Miguel tomó unas } \\
\text { cervezas }(20 \%)\end{array}$ & \\
\hline $\begin{array}{l}\text { Leticia se fumó } \\
\text { algunos cigarros }\end{array}$ & $74 \%$ & $\begin{array}{lrr}\text { Leticia } & \text { se } & \text { fumó } \\
\text { algunos } & \text { cigarrillos } \\
(6 \%) & \\
\end{array}$ & $\begin{array}{l}\text { Leticia fumó algunos } \\
\text { cigarros }(6 \%)\end{array}$ & $\begin{array}{l}\text { Leticia fumó } \\
\text { cigarros (14\%) }\end{array}$ \\
\hline
\end{tabular}

Cuadro 1. Resultados del test de juicio de gramaticalidad comentado en cada enunciado - Argentina.

Se observó, por medio de la comparación entre los datos presentes en el cuadro 1, que los enunciados "Miguel se tomó unas cervezas" y "Leticia se fumó algunos cigarros" obtuvieron, respectivamente, $80 \%$ y $74 \%$ de aceptación sin ninguna modificación, mientras que "Juan se comió algunas manzanas" y "María se bebió unos vasos de agua" obtuvieron, respectivamente, aceptación de $53 \%$ y $33 \%$ sin modificaciones.

Se percibe también que el único verbo que se ha sustituido por otro fue "beber", en $27 \%$ de las ocurrencias en las que se mantuvo el "se" télico y en $13 \%$ de las que se lo excluyó. En este caso, se sustituyó ese verbo por "tomar", aquel que, por lo que se puede ver en el cuadro 1, presenta una aceptación más amplia con el "se".

Se nota que la cantidad de aceptación de la combinación investigada en este trabajo no se restringe a los datos descritos en la columna dos, intitulada "Sin modificación", del cuadro 1. En realidad, los datos de la columna tres, intitulada "Mantenimiento del "se" télico y modificación en el enunciado", también se tratan de la aceptación de una oración que contiene el "se" télico combinado con un verbo cuyo complemento es introducido por un determinante plural indefinido, aunque el informante haya alterado el determinante presente en el enunciado por otro de la misma naturaleza. Así, la cantidad de aceptación de la combinación investigada con el verbo "beber" es de $60 \%$ y con el verbo "fumar" es de $80 \%$, como se observa en el cuadro 2 a continuación. 


\begin{tabular}{|l|l|l|}
\hline Enunciado & $\begin{array}{l}\text { Sin modificación o modificación con } \\
\text { mantenimiento del "se" télico y del } \\
\text { determinante plural indefinido }\end{array}$ & $\begin{array}{l}\text { Exclusión del célico con } \\
\text { mantenimiento del determinante } \\
\text { plural indefinido } \\
\text { modificación) }\end{array}$ \\
\hline $\begin{array}{l}\text { Juan se comió } \\
\text { algunas manzanas }\end{array}$ & $53 \%$ & $47 \%$ \\
\hline $\begin{array}{l}\text { María se bebió unos } \\
\text { vasos de agua }\end{array}$ & $60 \%$ & $40 \%$ \\
\hline $\begin{array}{l}\text { Miguel se tomó unas } \\
\text { cervezas }\end{array}$ & $80 \%$ & $20 \%$ \\
\hline $\begin{array}{l}\text { Leticia se fumó } \\
\text { algunos cigarros }\end{array}$ & $80 \%$ & $20 \%$ \\
\hline
\end{tabular}

Cuadro 2. Resumen de los resultados del test de juicio de gramaticalidad comentado en cada enunciado - Argentina.

Respecto a los resultados obtenidos por medio de la aplicación del test a hablantes nativos del español de Venezuela, en $97 \%$ de los enunciados objetivo (cincuenta y ocho enunciados), se mantuvo la combinación entre el "se" télico y verbos cuyos complementos eran introducidos por un determinante plural indefinido. En solo $3 \%$ de los enunciados objetivo (dos enunciados), se excluyó el "se" télico y se mantuvo el determinante plural indefinido.

El gráfico 2, que se presenta a continuación, se trata de un resumen de los resultados obtenidos por medio de la aplicación del test de juicio de gramaticalidad a hablantes nativos del español de Venezuela.

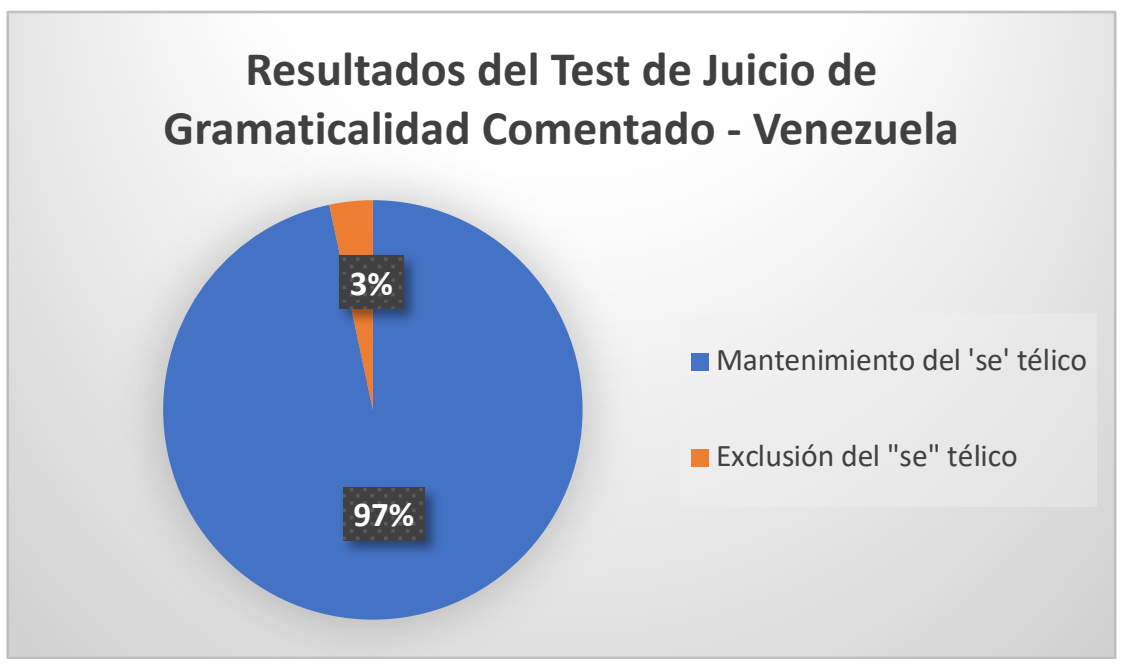

Gráfico 2. Resultados del test de juicio de gramaticalidad comentado - Venezuela.

Se observa que en $95 \%$ de los casos los participantes no hicieron modificaciones en los enunciados. Se las hizo solamente en uno, en el cual se sustituyó el verbo "beber" por "tomar", como se puede ver en el ejemplo (23), manteniéndose el "se" télico y el determinante plural indefinido.

(23) María se tomó unos vasos de agua. 
Por medio de la evaluación del juicio de gramaticalidad de los sujetos en cada enunciado del test separadamente, se observó que la aceptación de la combinación investigada en este trabajo era más amplia con algunos enunciados y más restringida con otros también en esa variedad, como se ve en el cuadro 3 a continuación.

\begin{tabular}{|c|c|c|c|}
\hline Enunciado & $\begin{array}{l}\text { Sin } \\
\text { modificación }\end{array}$ & $\begin{array}{l}\text { Mantenimiento del "se" télico } \\
\text { y modificación en el } \\
\text { enunciado }\end{array}$ & Exclusión del "se" télico \\
\hline $\begin{array}{l}\text { Juan se comió algunas } \\
\text { manzanas }\end{array}$ & $92 \%$ & & $\begin{array}{l}\text { Juan comió } \\
\text { manzanas }(8 \%)\end{array}$ \\
\hline $\begin{array}{l}\text { María se bebió unos } \\
\text { vasos de agua }\end{array}$ & $84 \%$ & $\begin{array}{l}\text { María se tomó unos vasos de } \\
\text { agua }(8 \%)\end{array}$ & $\begin{array}{l}\text { María bebió unos vasos de } \\
\text { agua }(8 \%)\end{array}$ \\
\hline $\begin{array}{l}\text { Miguel se tomó unas } \\
\text { cervezas }\end{array}$ & $100 \%$ & & \\
\hline $\begin{array}{l}\text { Leticia se fumó algunos } \\
\text { cigarros }\end{array}$ & $100 \%$ & & \\
\hline
\end{tabular}

Cuadro 3. Resultados del test de juicio de gramaticalidad comentado en cada enunciado - Venezuela.

Como se puede ver en el cuadro 3, los enunciados "Miguel se tomó unas cervezas" y "Leticia se fumó algunos cigarros" obtuvieron 100\% de aceptación, mientras que "Juan se comió algunas manzanas" y "María se bebió unos vasos de agua" obtuvieron, respectivamente, $92 \%$ y $84 \%$ de aceptación sin ninguna modificación.

Se observó también que, como en los resultados obtenidos en la aplicación del test a hablantes nativos del español de Argentina, los venezolanos también sustituyeron el verbo "beber" por "tomar", aunque en un porcentaje menor.

Se nota que la cantidad de aceptación de la combinación investigada en este trabajo no se restringe a los datos descritos en la columna dos, intitulada "Sin modificación", del cuadro 3. En realidad, los datos de la columna tres, intitulada "Mantenimiento del "se" télico y modificación en el enunciado", también se tratan de la aceptación de una oración que contiene el "se" télico combinado con un verbo cuyo complemento se introduce por un determinante plural indefinido, aunque el informante haya alterado el determinante presente en el enunciado por otro de la misma naturaleza. Así, la cantidad de aceptación de la combinación investigada con el verbo "beber" es de 92\%, como se observa en el cuadro 4 a continuación.

\begin{tabular}{|l|l|l|}
\hline Enunciado & $\begin{array}{l}\text { Sin modificación o modificación con } \\
\text { mantenimiento del "se" télico y del } \\
\text { determinante plural indefinido }\end{array}$ & $\begin{array}{l}\text { Exclusión del "se" télico con } \\
\text { mantenimiento } \\
\text { determinante plural indefinido }\end{array}$ \\
\hline $\begin{array}{l}\text { Juan se comió algunas } \\
\text { manzanas }\end{array}$ & $92 \%$ & $8 \%$ \\
\hline $\begin{array}{l}\text { María se bebió unos vasos de } \\
\text { agua }\end{array}$ & $92 \%$ & $8 \%$ \\
\hline Miguel se tomó unas cervezas & $100 \%$ & $0 \%$ \\
\hline $\begin{array}{l}\text { Leticia se fumó algunos } \\
\text { cigarros }\end{array}$ & $100 \%$ & $0 \%$ \\
\hline
\end{tabular}

Cuadro 4. Resumen de los resultados del test de juicio de gramaticalidad comentado en cada enunciado - Venezuela 


\section{ANÁLISIS}

Como se ha descrito en la sección anterior, los participantes de esta investigación, tanto hablantes nativos del español de Argentina como de Venezuela, juzgaron como naturales enunciados que contenían el "se" télico combinado con un verbo cuyo complemento era introducido por un determinante plural indefinido. Luego, se considera en este trabajo que dicha combinación es posible en ambas variedades.

Por lo tanto, no se ha refutado la hipótesis de que se puede usar el "se" télico combinado con verbos cuyos complementos sean introducidos por determinantes plurales indefinidos en el español de Argentina y de Venezuela, formulada con base en los resultados de Gomes y Martins (2020) sobre el español de España. Se puede comprender, entonces, que la propuesta de esos autores se extiende también a las variedades investigadas en este trabajo. Además, los resultados encontrados aquí son compatibles con los ejemplos creados y presentados por Suárez-Cepeda (2005) y Márquez (2020), en los que los autores combinaban el "se" télico con verbos que contenían complementos con determinantes plurales indefinidos.

Se debe discutir que, en los cuadros 2 y 4 , que se trataban de un resumen de los datos obtenidos en Argentina y Venezuela teniendo en cuenta el desempeño de los participantes en cada frase del test, la última columna podría permitir la refutación de la hipótesis del estudio, puesto que en ella se demuestra el porcentaje de exclusión del "se" télico en frases que contenían la presencia de un determinante plural indefinido. Sin embargo, en gran parte de las frases, la cantidad de aceptación fue más amplia que la de rechazo.

Es posible que los pocos datos de exclusión del "se" télico no se hayan motivado por su combinación con verbos cuyos determinantes eran introducidos por determinantes plurales indefinidos, sino por otros factores, como la frecuencia de uso de ese clítico con algunos verbos. Se observó en los resultados del test que la combinación del "se" télico con los verbos "tomar" y "fumar" tuvo mayor aceptación por los participantes de ambas variedades, mientras que su aceptación con "comer" y "beber" fue menor. Es posible que la asociación del "se" télico con los dos primeros verbos sea más frecuente que con los dos últimos.

Tanto en los datos de Argentina como en los de Venezuela, hubo casos en los que los participantes sustituyeron el verbo "beber" por el verbo "tomar". Eso puede también deberse a la frecuencia de la combinación del "se" télico con el segundo verbo. Martins, Gomes y 
Lourençoni (2017) ya señalaban en su estudio que la combinación del "se" télico con el verbo "tomar" es más frecuente en español, cuando comparado con otros verbos de ingestión 4 .

Al comparar las variedades investigadas, se observó que la aceptación de la combinación entre el "se" télico y verbos cuyos complementos eran introducidos por determinantes plurales indefinidos es más amplia en los resultados de los hablantes de la variedad de Venezuela, dado que se obtuvo 97\% de aceptación de las oraciones con "se" télico y determinantes plurales indefinidos en el complemento verbal, mientras que, en la variedad de Argentina, se obtuvo solamente $68 \%$ de la aceptación de esas oraciones, como se identifica, respectivamente, en los gráficos 2 y 1.

Teniendo en cuenta que la combinación investigada en este trabajo es posible en español, parece que las afirmaciones de Rothstein (2008) y Gomes y Martins (2020) de que determinantes plurales indefinidos conducen a una lectura télica son adecuadas. Para esos autores, no se deben interpretar los determinantes plurales indefinidos como aquellos en los que no hay delimitación, sino que como indicadores de la manera cómo se mide el complemento, lo que revela una expresión de cantidad que, en este caso, no es precisa.

Así, al retomar el continuum por medio del cual se pretendía explicar la relación entre el grado de definición del complemento y el valor aspectual télico, propuesto por Moure (1990), con el objetivo de discutir la posición que ocupan los determinantes plurales indefinidos, se ve que esos deben estar a la izquierda, dado que conducen a una lectura télica, como se observa en la imagen 2 a continuación.

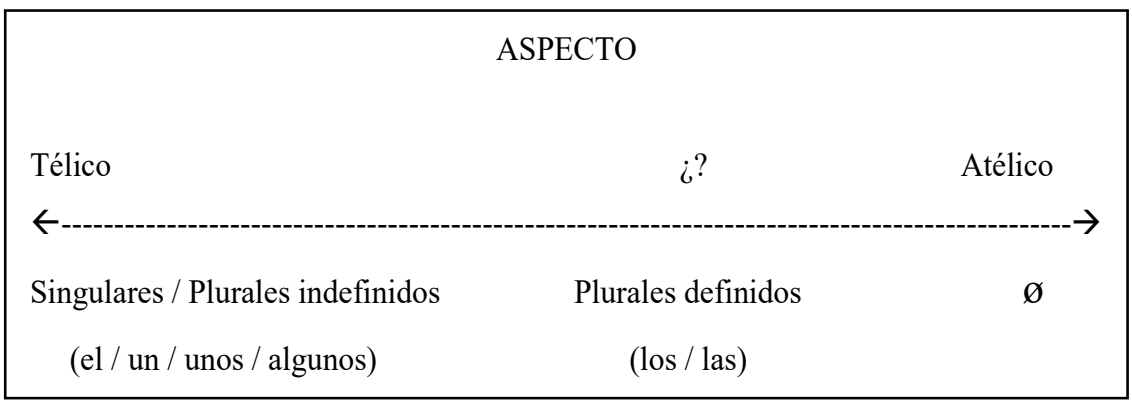

Imagen 2. Relación entre el grado de definición del complemento y el valor aspectual télico reformulada.

Aún así, es necesario verificar la contribución de los determinantes plurales definidos como "los(as)" para el valor aspectual de telicidad de la oración. De esa manera, será

4 Se presentan aquí dos interpretaciones posibles para la gran incidencia del "se" télico combinado con verbos de ingestión de manera general. La primera se ofrece por una de las pareceristas de este artículo, Teresa Cristina Wachowicz, a quien se le agradece la sugerencia. Según su interpretación, esa incidencia proviene de la idea de que verbos de ingestión, debido a su semántica inherente, puedan ofrecer un punto final a la situación, que sería el organismo del sujeto de la acción, acercándose, en este sentido, a un locativo. La segunda tiene como base la descripción de D’Introno, González y Rivas (2007), en la que se argumenta que el "se" con verbos de ingestión adquiere el valor semántico de beneficiario, en el que la acción se ejecuta a favor del referente del clítico. 
posible afirmar cuál es la posición que ocupan en el continuum, si se encuentran a la izquierda o a la derecha.

En este trabajo, se discutió la contribución que los determinantes plurales indefinidos tienen para el valor de telicidad cuando están en el complemento verbal. Sin embargo, parece relevante plantear la hipótesis de que esos determinantes puedan también desempeñar un papel importante cuando ocupan la posición de sujeto verbal. De acuerdo con autores como Verkuyl (2005), se transmiten también los valores de la telicidad por medio de otros ítems presentes en la oración además del complemento, como el sujeto.

Teniendo en cuenta que el sujeto sea importante en la transmisión del valor aspectual télico, es relevante cuestionar el papel que desempeñan los determinantes plurales indefinidos cuando ocupan la posición inicial de sujeto. En la propuesta de Verkuyl (2005), por ejemplo, el rasgo [ $₫$ SQA] del SN sujeto afecta la telicidad de la oración. Al integrar la propuesta de Verkuyl (2005) al análisis que se realiza en este trabajo y a la terminología que se adopta aquí, se entiende que la delimitación del determinante plural indefinido en el complemento verbal, que se sostuvo por los datos obtenidos en este artículo, podría también aplicarse cuando ese tipo de determinante introduce el sujeto. Así, el rasgo presente en este SN sujeto, que sería el [+SQA], sería responsable por volver la oración en télica, en el caso de oraciones que también presentan ese rasgo en el complemento de un verbo que sea dinámico.

De manera general, se nota que este trabajo contribuye a la comprensión de lo que caracteriza la telicidad, pues sus resultados señalan uno de los factores que colaboran en la transmisión del valor aspectual télico de una oración. Se sostiene que el valor de telicidad del "se" sea subespecificado. Así, es por medio de su concatenación con los otros elementos que componen la oración télica que se genera su especificación como un clítico aspectual. Además, se observó que la noción de delimitación, necesaria para la marcación lingüística del punto final de un evento, no depende de una cuantificación precisa del complemento.

\section{CONCLUSIÓN}

Se objetivó con este trabajo investigar si se puede usar el "se" télico en oraciones que contengan complementos verbales introducidos por determinantes plurales indefinidos en el español de Argentina y de Venezuela. Se predecía en la hipótesis del estudio, basada en Gomes y Martins (2020), que dicha combinación sería posible en las variedades investigadas.

Para este fin, se aplicó un test lingüístico de juicio de gramaticalidad comentado a quince hablantes nativos del español de Argentina y a quince hablantes nativos del español 
de Venezuela. Los resultados indicaron que, en ambas variedades, la combinación investigada es posible y, por eso, no se ha refutado la hipótesis.

Se observó que hubo una aceptación más amplia del "se" télico combinado con un verbo cuyo complemento era introducido por un determinante plural indefinido en la variedad de Venezuela, cuando comparada a la variedad de Argentina. Además, se percibió que, en ambas variedades, hubo una aceptación más amplia de la combinación investigada con los verbos "tomar" y "fumar".

Se ha concluido que determinantes plurales indefinidos conducen a una lectura télica de la oración. Se entiende, por lo tanto, que la delimitación de una oración, para que sea télica, no depende de la presencia de una cuantificación exacta del complemento, sino de un elemento que lo delimite.

Además, también se destaca que los resultados de este trabajo también contribuyen a la comprensión de los contextos en los que el uso del "se" télico es autorizado en español, puesto que este es un tema de investigación aún vigente en los estudios acerca del aspecto en esa lengua, como se señaló en la fundamentación teórica de este trabajo con base en Lourençoni y Martins (2017). Así, aunque no se presente aquí un panorama de todas las variedades del español, los datos de Argentina y de Venezuela, obtenidos en este estudio, combinados con los de España, obtenidos por Gomes y Martins (2020), sugieren que existe la posibilidad de que la combinación investigada en este trabajo sea gramatical también en otras variedades.

Se pretende, entonces, en etapas posteriores de esta investigación, verificar la frecuencia de uso de la combinación investigada en el habla espontánea e investigar si otros determinantes plurales, como los definidos "los(as)", y aquellos que reciben intensidad, por ejemplo "muchísimos", también conducen a una lectura télica de la oración.

Además, se considera relevante investigar el papel que los determinantes plurales desempeñan cuando ocupan la posición de sujeto para la transmisión del valor aspectual de telicidad. Por fin, se pretende verificar si esa combinación es posible también en otras variedades de la lengua española, además de la hablada en España, verificada en Gomes y Martins (2020), y en Argentina y Venezuela, verificada en este trabajo.

\section{AGRADECIMIENTOS}

Se agradece a la Fundação de Amparo à Pesquisa do Estado do Rio de Janeiro (FAPERJ) por la concesión de la beca de estudios Mestrado Nota 10 al primer autor de este trabajo durante la realización de la investigación y a las pareceristas de este artículo, Teresa Cristina Wachowicz, Roberta Pires de Oliveira y Raquel Fellet Lawall, por los 
comentarios y sugerencias que han hecho. Los errores presentes en el texto son de entera responsabilidad de los autores.

\section{REFERÊNCIAS}

BASSO, Renato Miguel. Telicidade e Detelicização. Revista Letras, v. 72, n.1, p. 215-232, 2007.

BERTINETTO, Pier Maco. On a frequent misunderstanding in the temporal-aspectual domain: the perfectivetelic confusion. In: CECCHETTO, Carlo; CHIERCHIA, Gennaro; GUASTI, Maria Teresa. Semantic interfaces: reference, anaphora and aspect. Stanford: CSLI, 2001, p. 177-210.

CHAUDRON, Craig. Data Collection in SLA Research. In: DOUGHTY, Catherine; LONG, Michael. The Handbook of Second Language Acquisition. Malden, MA: Blackwell Publishing, 2003, p. 762-828.

CELERI, Waniston Coelho. A composicionalidade aspectual revisitada. 2008. Disertación (Máster em Lingüística) - Faculdade de Letras, Universidade Federal do Rio de Janeiro, Rio de Janeiro, 2008.

CHOMSKY, Noam. Syntactic structures. The Hague: Mouton, 1957.

COMRIE, Bernard. Aspect: an introduction to the study of verbal aspect and related problems. Cambridge: Cambridge University Press, 1976.

DAHL, Östen. Logic, Pragmatic and Grammar. Gotemborg: University of Göteborg, Departament of Linguistics, 1977.

DE MIGUEL, Elena. El aspecto léxico. In: BOSQUE, Ignacio; DEMONTE, Violeta. Gramática Descriptiva de la lengua española. Madrid: Espasa Calpe, 1999, p. 2977-3060.

DE MIGUEL, Elena; LAGUNILLA, Marina Fernández. El Operador Aspectual Se. Revista Española de Lingüística, v. 30, n. 1, p. 13-43, 2000

DECLERCK, Renaat. Aspect and bounded/unbounded (telic/atelic) distinction. Linguistics, London, n. 17, n. 1, p. 761-794, 1979

D'INTRONO, Francesco; GONZALEZ, Verónica; RIVAS, Javier. Aspectos sintácticos y semánticos del pronombre SE. Boletín de Lingüística, v. 19, n. 28, p. 5-25, 2007.

FONTANELLA DE WEINBERG, María Beatriz. El Español de la Argentina y sus variedades regionales. Bahía Blanca: Asociación Bernardino Rivadavia, 2004.

GASS, Susan. Language transfer and universal grammatical relations. Language Learning, v. 29, n.1, p. 327 344, 1979. DOI https://doi.org/10.1111/j.1467-1770.1979.tb01073.x. Accesado en: 19 de marzo de 2020.

GASS, Susan. An investigation of syntactic transfer in adult second language learners. In: SCARCELLA, Robin; KRASHEN, Stephen. Research in Second Language Acquisition. Rowley, MA: Newbury House, 1980, p. 132-141.

GOMES, Jean Carlos da Silva. Telicidade e sua compatibilidade com expressões adverbiais durativas no espanhol. 2017. Monografía (Graduación en Letras Portugués - Español) - Faculdade de Letras, Universidade Federal do Rio de Janeiro, Rio de Janeiro, 2017.

GOMES, Jean Carlos da Silva. O comprometimento do aspecto perfect na Doença de Alzheimer. 2020. Disertación (Máster en Lingüística) - Faculdade de Letras, Universidade Federal do Rio de Janeiro, Rio de Janeiro, 2020

GOMES, Jean Carlos da Silva; MARTINS, Adriana Leitão. Telicidade e determinantes plurais indefinidos no espanhol da Espanha.Domínios da Lingu@gem, v. 14, n. 2, p. 482-509, 2020. DOI https://doi.org/10.14393/DL42-v14n2a2020-6. Accesado en: 19 de marzo de 2020. 
GORDON, Peter. The truth-value judgment task. In: MCDANIEL, Dana; MCKEE, Cecile; CAIRNS, Helen Smith. Methods for assessing children's syntax. Cambridge: The MIT Press, 1996, p. 211-232.

GRÉGIS, Rosi Ana. Testes de julgamento gramatical em pesquisas de aquisição de segunda língua. 2007. Tesis (Doctorado en Letras). Faculdade de Letras - Pontifícia Universidade Católica do Rio Grande do Sul, Rio Grande do Sul, 2007.

GROLLA, Elaine. Metodologias experimentais em aquisição da linguagem. Revista Estudos da Lingua(gem), v. 7, n.1, p. 9-42, 2009. DOl https://doi.org/10.22481/el.v7i2.1090. Accesado en: 19 de marzo de 2020.

HAVIK, Else; ROBERTS, Leah; VAN HOUT, Roeland; SCHREUDER, Robert; HAVERKORT, Marco. Processing subject-object ambiguities in the L2: A self-paced reading study with German L2 learners of Dutch. Language Learning, v. 59, n.1, p. 73-112, 2009. DOI https://doi.org/10.1111/i.1467-9922.2009.00501.x. Accesado en: 13 de mayo de 2020

KRIFKA, Manfred. Varieties of semantic evidence. In: MAIENBORN, Claudia; VON HEUSINGER, Klaus; PORTNER, Paul. Semantics: an international handbook of natural language meaning. Berlin: Mouton de Gruyter, 2011, p. 242-267.

LICERAS, Juana. M. The role of intake in the determination of learners' competence. In: GASS, Susan; MADDEN, Carolyn. Input in Second Language Acquisition. Rowley, MA: Newbury House, 1985, p. 354-373.

LIGHTBOWN, Patsy; SPADA, Nina; WHITE, Lydia. The role of instruction in second language acquisition. Studies in Second Language Acquisition, v. 15, n. 2, p. 143-145, 1993. DOI https://doi.org/10.1017/S027226310001192X. Accesado en: 19 de marzo de 2020

LÓPEZ, Cristina Sánchez. Las construcciones con se: estado de la cuestión. In: LÓPEZ, Cristina Sánchez. Las construcciones con se. Madrid: Visor Libros, 2002, p. 18-167.

LOURENÇONI, Débora Cristina Paz Paz. Telicidade e sua realização pelo operador aspectual se no espanhol. 2017. Disertación (Máster en Lingüística) - Faculdade de Letras, Universidade Federal do Rio de Janeiro, Rio de Janeiro, 2017

LOURENÇONI, Débora Cristina Paz Paz. O traço de telicidade e suas realizações no português do Brasil e no espanhol do Chile. 2014. Monografía (Graduación en Letras Portugués - Español) - Faculdade de Letras, Universidade Federal do Rio de Janeiro, Rio de Janeiro, 2014

LOURENÇONI, Débora Cristina Paz Paz; MARTINS, Adriana Leitão. O traço aspectual de telicidade e suas realizações no português do Brasil e no espanhol do Chile. SEDA - Revista de Letras da Rural/RJ, v. 1, n. 2, p. 5-28, 2016

LOURENÇONI, Débora Cristina Paz Paz; MARTINS, Adriana Leitão. O operador aspectual se no espanhol do Chile: contextos semânticos e morfossintáticos de uso. Fórum Linguístico, v. 14, n. 2, p. 2076-2095, 2017. DO http://dx.doi.org/10.5007/1984-8412.2017v14n2p2076. Accesado em: 13 de mayo de 2020.

MÁRQUEZ, Pablo Devís. Estatividad, transitividad y clíticos. Nueva Revista de Filología Hispánica, v. 73, n. 1, p. 3-46, 2020. DOl https://doi.org/10.24201/nrfh.v68i1.3581. Accesado en: 25 de enero de 2020.

MARTINS, Adriana Leitão. A desintegração de tempo na demência do tipo Alzheimer. 2010. Tesis (Doctorado en Lingüística). Faculdade de Letras, Universidade Federal do Rio de Janeiro, Rio de Janeiro, 2010.

MARTINS, Adriana Leitão:; GOMES, Jean Carlos da Silva; LOURENCONI, Débora Cristina Paz Paz. Telicidade e expressões adverbiais durativas no espanhol da Espanha: uma análise a partir do se télico. Caderno de squibs: temas em estudos formais da linguagem, v. 3, n. 1, p. 1-15, 2017

MOURE, Teresa. El contenido aspectual telicidad en las cláusulas biactanciales del español. Verba, n. 18, n. 1, p. 353-374, 1990.

ROLDÁN, Mercedes. Spanish constructions with se. Language Sciences, v. 18, n. 1, p. 15-29, 1971.

ROTHSTEIN, Susan. Theoretical and crosslinguistic approaches to the semantics of aspect. Amsterdam: Benjamins, 2008.

SANZ, Monserrat. Events and predication: a new approach to syntactic processing in English and Spanish. Amsterdam: John Benjamins B. V., 2000 
SANZ, Monserrat; LAKA, Itziar. Oraciones transitivas con se: El modo de acción en la sintaxis. In: LÓPEZ, Cristina Sánchez. Las construcciones con se. Madrid: Visor Libros, 2002, p. 309-336.

SLABAKOVA, Roumyana. L1 Transfer revisited the L2 Acquisition of telicity marking in English by Spanish and Bulgarian native speakers. Linguistics, n. 38, v. 4, p. 739-770, 2000. DOI https://doi.org/10.1515/ling.2000.004. Accesado en: 12 de junio de 2020.

SUÁREZ CEPEDA, Sonia. Pedro comió la torta vs. Pedro se comió la torta: L2 Acquisition of Spanish Telic se constructions. Anvario, n. 7, v. 1, p. 277-295, 2005.

VERKUYL, Henk. Aspectual composition: surveying the ingredients. In: VERKUYL, Henk; SWART, Henriette; VAN HOUT, Anne. Perspectives on aspect. Dordrecht: Springer, 2005, p. 19-39.

WACHOWICZ, Teresa Cristina. Telicidade e classes aspectuais. Revista do Gel, v. 5, n. 1, p. 57-68, 2008

WILSON, Stephen; HENRY, Maya; BESBRIS, Max; OGAR, Jennifer; DRONKERS, Nina; JARROLD, William; MILLER, Bruce; GORNO-TEMPINI, Maria Luisa. Connected speech production in three variants of primary progressive aphasia. Brain, v. 133, n. 1, p. 2069-2088, 2010. DOI https://doi.org/10.1093/brain/awq129. Accesado en: 06 de noviembre de 2019.

ZOBL, Helmut. Sources of linguistic knowledge and uniformity of nonnative performance. Studies in Second Language Acquisition, v. 14, n.1, p. 387-403, 1992. DOI https://doi.org/10.1017/S0272263100011190. Accesado en: 19 de marzo de 2020 